005.5

EARLY IMPACTS OF THE COVID-19 PANDEMIC ON SEXUAL BEHAVIOUR IN BRITAIN: FINDINGS FROM A LARGE, QUASI-REPRESENTATIVE SURVEY (NATSALCOVID)

${ }^{1} \mathrm{C}$ Mercer* ${ }^{1,2 S}$ Clifton, ${ }^{3}$ Riddell, ${ }^{4} \mathrm{C}$ Tanton, ${ }^{1} \mathrm{P}$ Sonnenberg, ${ }^{1} \mathrm{~A}$ Copas, ${ }^{3} \mathrm{R}$ Boso Perez, ${ }^{4} \mathrm{~W}$ Macdowell, ${ }^{1} \mathrm{D}$ Menezes, ${ }^{1} \mathrm{E}$ Dema, ${ }^{1} \mathrm{~L}$ Freeman, ${ }^{1} \mathrm{M}$ Ridge, ${ }^{4} \mathrm{C}$ Bonell, ${ }^{1} \mathrm{~N}$ Field, ${ }^{3} \mathrm{~K}$ Mitchell. ${ }^{1}$ University College London, London, UK; ${ }^{2}$ Natcen Social Research, London, UK; ${ }^{3}$ University of Glasgow, Glasgow, UK; ${ }^{4}$ London School of Hygiene and Tropical Medicine, London, UK

\subsection{6/sextrans-2021-sti.78}

Background COVID-19 has impacted all aspects of life, including people's sex lives, via experience of the disease and measures to prevent transmission. We examined sexual behaviour in Britain during the initial national 'lockdown' $(\geq 23 / 3 / 2020)$ and compared this to the 3 months pre-lockdown.

Methods We analysed weighted web-panel survey data from a quota-based sample of 6,654 people in Britain. The questionnaire, fielded 29/7-10/8/2020, included questions about sexual activities pre- and during lockdown, and perceived changes in frequency between these timeframes. We used descriptive statistics and multivariable regression to examine independent associations with relationship status, age, gender, and health.

Results Altogether, 91.2\% of sexually-experienced participants reported any sexual activity during lockdown; $85.7 \%$ reporting 'in-person'/physical partnered activities. Around half reported no change in frequency of partnered-sex versus prelockdown, however, those not cohabiting were more likely than those cohabiting to report changes $(75.6 \%$ versus $35.1 \%)$ - typically declines. Masturbation (62.0\%) and virtual/digital activities $(54.3 \%)$ were less commonly reported during lockdown, although they were more commonly reported in those not cohabiting versus cohabiting $(69.2 \%$ versus $57.9 \%, 67.4 \%$ versus $46.7 \%$, respectively). Changes in reported frequency of virtual/digital activities were more common (66.4\%) than inperson activities, with increases as likely as declines, except for porn use, where twice as many perceived an increase than a decrease. After adjustment, those reporting a decline in sex were more likely to be: non-cohabiting (AOR:1.68,95\% CI:1.45-1.95), aged <25 years (AOR:1.99,1.57-2.51), male (AOR:1.17,1.02-1.35), to report depressive/anxiety symptoms (AOR:1.63,1.41-1.89) or COVID symptoms/diagnosis (AOR:1.24,1.01-1.52).

Conclusions Most people reported some form of sex during lockdown and around half had not experienced changes in partnered-sex compared to pre-lockdown. However, considerable differences existed for certain populations (e.g. young people) that may exacerbate, or be exacerbated by, COVID19's wider detrimental effects on physical and mental health. This potential intersectionality needs consideration when designing individual and public health interventions.

\section{O05.6 SEXUAL CONTACT WITH PARTNERS OUTSIDE OF HOUSEHOLD DURING THE COVID-19 PANDEMIC: INVESTIGATING MOTIVATIONS AND DECISION-MAKING USING NATSAL-COVID DATA}

${ }^{1} \mathrm{~K}$ Maxwell ${ }^{*},{ }^{2} \mathrm{~L}$ Freeman, ${ }^{1} \mathrm{R}$ Boso Perez, ${ }^{2} \mathrm{D}$ Reid, ${ }^{2} \mathrm{D}$ Menezes, ${ }^{2} \mathrm{P}$ Sonnenberg, ${ }^{2} \mathrm{C}$ Mercer, ${ }^{1} \mathrm{~K}$ Mitchell, ${ }^{2} \mathrm{~N}$ Field. ' University of Glasgow, Glasgow, UK; ${ }^{2}$ University College London (UCL), London, UK
Background Worldwide, efforts to control SARS-CoV-2 transmission have included lockdowns and restrictions on contact with others, including sexual partners. Our research (NatsalCOVID) indicates that in the UK, $10 \%$ of people aged 18-59 had physical or sexual contact with a romantic or sexual partner outside their household $(\mathrm{PCOH})$ during a period in which contact was limited. We explored motivations and decisionmaking among people reporting $\mathrm{PCOH}$ in the four months following the initial national lockdown on 23rd March 2020.

Method Semi-structured interviews were conducted with 18 individuals reporting $\mathrm{PCOH}$ during a period in which physical distancing measures were in place. Participants were recruited through a large, quasi-representative survey investigating sexual behaviour in the UK during the COVID-19 pandemic (NatsalCOVID). Interviews were analysed thematically.

Results Participants were single $(n=8)$ or in long-term, noncohabiting, relationships $(n=10)$. While participants in the two groups differed in their reported motivations for $\mathrm{PCOH}$, all demonstrated complex and individualised decision-making, weighing up risks such as SARS-CoV-2 transmission, judgement of peers, and benefits, including feelings of security and improved mental health. For those in relationships, the primary motivation was continuity: participants expected to continue seeing their romantic partner. Participants rationalised this contact as 'low risk' in relation to other 'risks' of COVID-19 exposure, and reduced other activities (such as shopping, seeing friends) to maintain this contact. For single participants, loneliness and boredom were reported as the primary motivators for $\mathrm{PCOH}$, with dating apps often used to facilitate contact. For both groups, evidence of considered decision-making was clear, with participants referencing government guidance, personal situations, and risk when describing their deliberations.

Conclusion Individuals did not make decisions about $\mathrm{PCOH}$ lightly. However, physical contact with partners was considered important and thus rationalised. Public health policy-makers must therefore consider sexual behaviour and needs for physical contact in designing effective future public health messaging.

\section{Syphilis clinical}

\subsection{ORAL AND ANAL TREPONEMA PALLIDUM DETECTION IN MEN WHO HAVE SEX WITH MEN WITH EARLY INFECTIOUS SYPHILIS: A CROSS-SECTIONAL STUDY}

\footnotetext{
${ }^{1,2}$ J Towns*, ${ }^{3} \mathrm{D}$ Leslie, ${ }^{1}$ I Denham, ${ }^{1} \mathrm{R}$ Wigan, ${ }^{3} \mathrm{~F}$ Azzato, ${ }^{4,5,6} \mathrm{D}$ Williamson, ${ }^{5} \mathrm{D}$ Lee, ${ }^{1,2,8} \mathrm{E}$ Chow, ${ }^{1,2} \mathrm{C}$ Fairley, ${ }^{7} \mathrm{~S}$ Graves, ${ }^{1,2,9,10} \mathrm{~L}$ Zhang, ${ }^{1,2} \mathrm{M}$ Chen. ${ }^{1}$ Melbourne Sexual Health Centre, Melbourne, Australia; ${ }^{2}$ Central Clinical School, Faculty of Nursing, Medicine and Health Sciences, Monash University, Melbourne, Australia; ${ }^{3}$ Victorian Infectious Diseases Reference Laboratory, The Peter Doherty Institute for Infection and Immunity., Melbourne, Australia; ${ }^{4}$ Microbiological Diagnostic Unit Public Health Laboratory, Department of Microbiology and Immunology, The University of Melbourne at The Peter Doherty Institute for Infection and Immunity., Melbourne, Australia; ${ }^{5}$ Department of Microbiology and Immunology, The University of Melbourne at The Peter Doherty Institute for Infection and Immunity., Melbourne, Australia; ${ }^{6}$ Department of Microbiology, Royal Melbourne Hospital., Melbourne, Australia; ${ }^{7}$ Australian Rickettsial Reference Laboratory, Barwon Health. Geelong, Australia; ${ }^{8}$ Centre for Epidemiology and Biostatistics, Melbourne School of Population and Global Health, The University of Melbourne., Melbourne, Australia; ${ }^{9}$ ChinaAustralia Joint Research Center for Infectious Diseases, School of Public Health, Xi'an Jiaotong University Health Science Center., Xi'an, China; ${ }^{10}$ Department of Epidemiology and Biostatistics, College of Public Health, Zhengzhou University., Zhengzhou, China
} 